\title{
FORMAÇÃO DE RECURSOS HUMANOS EM VIGILÂNCIA EM SAÚDE AMBIENTAL: UMA PROPOSTA DE AVALIAÇÃO.
}

\author{
RIO DE JANEIRO/RJ JULHO/2018
}

\author{
Ivisson Carneiro Medeiros da Silva - LABEAD/IESC/UFRJ - ivisson@iesc.ufrj.br \\ Carmen Ildes Rodrigues Froes Asmus - FM/UFRJ - carmenfroes@iesc.ufr.br
}

Tipo: Investigação Científica (IC)

Natureza: Relatório Final de Pesquisa

Categoria: Pesquisa e Avaliação

Setor Educacional: EDUCAÇÃO CONTINUADA EM GERAL

\begin{abstract}
RESUMO
A Vigilância em Saúde Ambiental (VSA) pode ser definida como um conjunto de ações propostas pelos diversos setores da sociedade, abrangendo as diversas áreas do conhecimento, com o objetivo de permitir a observação e análise das alterações ambientais que interferem na saúde humana. Desde a implantação da área de Vigilância em Saúde Ambiental no país pela Fundação Nacional de Saúde (FUNASA), suas atividades foram centradas na capacitação de recursos humanos e na estruturação dos Sistemas de informação para subsidiar as decisões desta área. O Programa de formação de recursos humanos em vigilância em saúde ambiental do Instituto de Estudos em Saúde Coletiva da Universidade Federal do Rio de Janeiro (IESC/UFRJ), parte integrante da Universidade Aberta do SUS (UNA-SUS) produz cursos de capacitação para auxiliar no desenvolvimento e formação dos trabalhadores do SUS. O Programa ofereceu três cursos de capacitação à distância, no período de 2010 a 2013 nas áreas de vigilância da qualidade da água para consumo humano, avaliação de risco a saúde humana por exposição a substâncias químicas e Saúde, desastres e desenvolvimento com a oferta de 1.000 vagas em cada curso. Este trabalho tem por objetivo avaliar o Programa para a qualificação das ações na área da Vigilância em Saúde Ambiental no Brasil. O estudo foi desenvolvido em duas etapas. Na primeira etapa foi feita uma análise descritiva dos dados sobre o perfil sociodemográfico dos aprovados. Na segunda etapa empregou-se um questionário semiestruturado para analisar os resultados do Programa. Profissionais de todos os estados e 517 municípios foram capacitados em um ou mais cursos oferecidos. Do total de 1.604 alunos capacitados, 215 (13,2\%) responderam ao questionário sendo que 98,7\% afirmaram que os cursos oferecidos ampliaram suas competências em vigilância em saúde ambiental e 59,4\% relataram como ótima, a experiência em EAD, mostrando a importância deste metodologia para capacitação profissional em nível nacional.
\end{abstract}

Palavras-chave: Saúde ambiental. Educação a distância. Formação de recursos humanos.

\section{AGRADECIMENTOS}

À REDE UNA-SUS PELA PARCERIA PARA A IMPLANTAÇÃO DO LABORATÓRIO DE EDUCAÇÃO À DISTÂNCIA, A CGVAM/MS PELO APOIO FINANCEIRO PARA DESENVOLVIMENTO DO PROJETO E A TODOS QUE PARTICIPARAM DO ESTUDO. 


\section{1 - Introdução}

A Vigilância em Saúde Ambiental (VSA) é uma área da vigilância em saúde definida por Tambellini e Câmara (1998) como o campo da saúde coletiva que tem como característica sua interface com todas as Vigilâncias do setor saúde, além da articulação transversal com atores de diversos setores que extrapolam a área de saúde, como o meio ambiente, a defesa civil, a agricultura, etc. Diversas áreas do conhecimento como Epidemiologia, Ciências Sociais, Toxicologia, entre outras, compõem o arcabouço teórico que permite a identificação, análise e proposição de soluções para as situações de degradação ambiental provocadas pelo homem, através do seu modelo de produção e que, muitas vezes, provocam efeitos à saúde humana. A VSA no Brasil foi implantada através da Fundação Nacional de Saúde (BRASIL, 2000) tendo, entre outras competências, a gestão do subsistema nacional de vigilância ambiental. A partir da criação da Coordenação de Vigilância em Saúde Ambiental, e posteriormente, do Departamento de Vigilância em Saúde Ambiental e de Saúde do Trabalhador, no âmbito da Secretaria de Vigilância em Saúde do Ministério da Saúde, a área adquiriu reconhecimento institucional, com representações em todos os Estados. Essa descentralização promoveu uma forma nova de atuação no SUS contribuindo para sua consolidação. A prática da vigilância em Saúde Ambiental contribui para a identificação e interpretação das vulnerabilidades socioambientais que pertencem a uma complexa estrutura de fatores determinantes do processo saúde - doença (ROHLFS et al., 2011). O acentuado processo de degradação ambiental originado na década de 60 , com a maior utilização de processos produtivos poluentes, e uso de substâncias químicas nocivas à saúde humana, nos países em desenvolvimento como o Brasil, foi a condição desencadeante para o início das ações deste campo de Vigilância. A implantação de políticas de capacitação dos quadros técnicos da área tornou-se essencial para possibilitar a identificação de possíveis situações de risco ambiental à saúde humana, de forma precoce, assim como permitir a realização de ações de intervenção e remediação. Esses profissionais, oriundos de formações e graus de especialização diversos, necessitam ampliar o espectro de conhecimentos de que dispõem para que atuem ativamente na vigilância, tendo por base a interdisciplinaridade dos problemas ambientais que podem ter efeitos lesivos sobre a saúde das comunidades expostas (CÂMARA \& TAMBELLINI, 2003). Diversos fatores ambientais determinam desfechos deletérios à saúde humana. O Brasil é o maior consumidor de agrotóxicos do mundo e diversos estudos identificam efeitos adversos dessas substâncias no processo saúdedoença, como prematuridade e baixo peso e aumento do risco de câncer (GUIMARÃES et. al., 2014; KRAWCZYK et.al., 2016). Outras questões ambientais antropogênicas como as queimadas e a poluição ambiental têm impactos sobre a saúde humana. Amâncio e Nascimento (2012) estudaram a associação entre o número de internações 
por asma brônquica na infância e a exposição a material particulado, dióxido de enxofre e ozônio, encontrando associação estatisticamente significativa nas análises envolvendo os dois primeiros poluentes. Asmus et al. (2016) em uma revisão sistemática sobre a exposição de crianças à poluentes ambientais no Brasil, identificaram 74 artigos publicados, entre 1995 e 2015, que apontavam uma associação entre a exposição a agrotóxicos, diversos metais (mercúrio, chumbo, arsênio, cádmio, etc.) e poluentes do ar e efeitos deletérios na saúde das crianças estudadas. Cada ente federativo do Estado brasileiro tem a responsabilidade de incluir a vigilância em saúde ambiental em sua estrutura organizacional de Governo. Por outro lado, a VSA não se encontra implementada em todos os municípios do País. Além disso, onde já se encontra em funcionamento, verifica-se a necessidade de fortalecimento das ações de capacitação dos profissionais que nela atuam. Para isto, a formação e qualificação de recursos humanos em grande escala se faz necessária (ROHLFS et al., 2011). Este trabalho objetiva apresentar a experiência desenvolvida pelo Laboratório de Educação à Distância (Labead) do IESC/UFRJ, na capacitação de profissionais do sistema público de saúde e ambiente de todo o país. Desta forma, pretende contribuir para a ampliação do conhecimento relativo ao uso das tecnologias e ferramentas vinculadas ao ensino à distância, como uma estratégia para a formação de recursos humanos a nível nacional.

\section{1 - O Programa de Formação de Recursos Humanos em Vigilância em Saúde Ambiental do IESC/UFRJ.}

O Programa de formação de recursos humanos em vigilância em saúde ambiental foi criado em 2010, a partir de um projeto aprovado pelo Ministério da Saúde, com financiamento do Fundo Nacional de Saúde, e integra o Laboratório de Educação a distância do IESC/UFRJ. Tem como uma de suas atividades a elaboração de cursos de especialização e capacitação à distância para trabalhadores de nível superior, vinculados ao sistema público de saúde e órgãos afins, no campo de conhecimentos e práticas que engloba a área de Vigilância em Saúde Ambiental, qualificando quadros técnicos em todo o país e auxiliando a consolidação deste campo de atuação no território nacional. Os cursos de capacitação online compreendidos por este Programa, com carga horária aproximada de 100 horas, foram produzidos por pesquisadores especialistas nos temas propostos, do próprio IESC e de outras instituições de ensino e pesquisa, em conjunto com profissionais diretamente envolvidos na gestão das ações referentes a estes temas. Esses profissionais reunidos definiram o conteúdo e a produção do material didático. Os textos foram adaptados para a metodologia da educação à distância e disponibilizados aos alunos em um ambiente virtual de aprendizagem - Moodle. Freire (1996) observou que quanto mais discussões existam entre os sujeitos, mais possibilidades de novos aprendizados. Assim sendo, estes cursos foram elaborados tendo como proposta a formação autônoma do aluno, onde 
este é agente ativo, construindo seu saber em discussões apresentadas pelo professortutor, que atua como estimulador e orientador do aprendizado sobre os temas propostos. No período de 2010 a 2013 foram elaborados três cursos de capacitação, abrangendo os conteúdos teóricos e práticos relacionados à Vigilância da qualidade da água para Consumo humano, Avaliação de risco à saúde humana por exposição a substâncias químicas e Saúde, Desastres e Desenvolvimento. Foram abertas 3.000 vagas (1.000 vagas para cada curso) para profissionais de nível superior vinculados ao setor público de saúde e ambiente, à nível municipal, estadual e federal. Um total de 4.901 profissionais se candidataram às vagas oferecidas, sendo selecionados de acordo com os critérios estabelecidos pela coordenação técnica, quais sejam: nível superior, atuação no sistema único de saúde e na área de vigilância em saúde (DA SILVA et al., 2014). 2 Metodologia Trata-se de um estudo observacional transversal onde a população de estudo foi constituída por 1604 alunos aprovados em cada um dos cursos ofertados no período entre 2010 e 2013. Dados fornecidos no momento da inscrição no processo seletivo foram relacionados com os resultados dos alunos aprovados em cada curso. Análises descritivas sobre cada um dos cursos foram realizadas a partir das seguintes variáveis: distribuição por gênero, município, estado e região do país, Esfera de atuação e formação acadêmica. Foi elaborado um mapa com a distribuição espacial dos alunos no território nacional para auxiliar na observação da abrangência dos cursos. Estas imagens foram construídas a partir dos dados do código de endereçamento postal (CEP) informado pelos alunos na ficha de inscrição através do aplicativo google maps. Além do estudo estatístico descritivo, foi enviado para cada um dos alunos aprovados um questionário para avaliarmos a percepção dos mesmos sobre o processo de aprendizagem e sobre a metodologia da EAD para o aprimoramento dos conhecimentos para execução das suas atividades laborais. Essas respostas foram categorizadas a partir da escala de Likert (1903 - 1981) para medir atitudes e opiniões sobre as questões nas quais os indivíduos especificam seu grau de concordância ou discordância com cada item perguntado (BECKER, 2015). 3 - Resultados Todas as 3.000 vagas oferecidas nos cursos foram preenchidas por profissionais de todas as regiões do país, com um total de $1.604(53,8 \%)$ indivíduos capacitados. Vale observar que 50 alunos se inscreveram e foram aprovados nos três cursos, 181 em dois cursos e 1.382 em apenas um curso, significando um total de 1.894 aprovações $(63,1 \%$ das 3.000 vagas ofertadas). A maioria dos alunos era do sexo feminino (70\%) e tinha formação universitária na grande área das ciências da saúde (576 - 33\%) e ciências biológicas (383 - 22\%). Outras áreas que tiveram grande número de alunos foram as ciências agrárias (157 - 9\%), área que abriga profissionais da medicina veterinária, além das diversas ramificações das engenharias (155 - 9\%) e das ciências sociais aplicadas (134 - 8\%). O Programa abrangeu profissionais de todos os estados brasileiros, porém, apenas 517 (9,3\%) municípios de todo o país foram alcançados. As regiões Sudeste, 
Nordeste e Sul, tiveram o maior número de alunos concluintes. Houve maior participação de alunos das áreas metropolitanas e da região centro-sul do Brasil, oriundos dos Estados mais acometidos por desastres ambientais e maiores áreas de solo contaminado. A tabela 1 apresenta a distribuição dos alunos de acordo com a esfera de governo em que exerciam suas atividades profissionais. Em todos os 3 cursos oferecidos 999 (52,7\%) alunos trabalhavam no nível municipal, 481 (25,4\%) em nível estadual e $217(11,5 \%)$ trabalhavam no nível federal. Alguns alunos preencheram as fichas com a informação de outro tipo de vínculo e 176 não preencheram esta informação, o que determinou uma perda de 9,3\% nesta variável.

Tabela 1: Distribuição dos alunos aprovados por curso e tipo de vínculo
institucional. Rio de janeiro. 2010 a 2014
\begin{tabular}{lcccccccc}
\hline \multicolumn{7}{c}{ Cursos } \\
\hline Tipo de vínculo & ARSH & $(\%)$ & DESASTRES & $(\%)$ & AGUA & $(\%)$ & TOTAL & $(\%)$ \\
\hline Municipal & 354 & $54,2 \%$ & 240 & $39,6 \%$ & 405 & $63,8 \%$ & 999 & $52,7 \%$ \\
Estadual & 198 & $30,3 \%$ & 123 & $20,3 \%$ & 160 & $25,2 \%$ & 481 & $25,4 \%$ \\
Federal & 73 & $11,2 \%$ & 77 & $12,7 \%$ & 67 & $10,6 \%$ & 217 & $11,5 \%$ \\
Outro & 21 & $3,2 \%$ & 0 & $0,0 \%$ & 0 & $0,0 \%$ & 21 & $1,1 \%$ \\
Sem resposta & 7 & $1,1 \%$ & 166 & $27,4 \%$ & 3 & $0,5 \%$ & 176 & $9,3 \%$ \\
\hline Total & 653 & $100,0 \%$ & 606 & $100,0 \%$ & 635 & $100,0 \%$ & 1894 & $100,0 \%$ \\
\hline
\end{tabular}

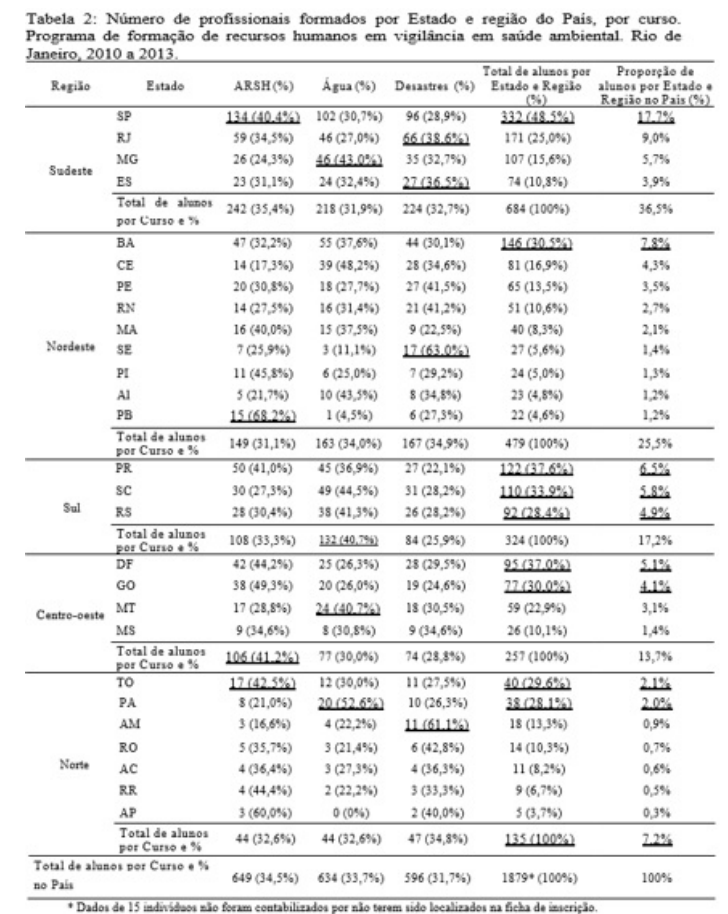

Ao apresentar os dados dos municípios com profissionais capacitados por estado sob a ótica dos 517 municípios alcançados com a participação de pelo menos um profissional, observa-se que os 88 municípios do Estado de São Paulo correspondem a 17\% do total de municípios com profissionais capacitados por este Programa. Os municípios do Estado da Bahia corresponderam a 9,5\% (49) do total de municípios com pelo menos um profissional concluinte dos cursos oferecidos. A região Nordeste, apesar de ser a região com menor abrangência regional, $7,7 \%$ do total de municípios da região com um ou mais profissionais capacitados, corresponde a 26,9\% (139) dos 517 municípios 
alcançados pelo Programa. Os 119 municípios da região Sul correspondem a $23 \%$ do total de municípios atendidos e as regiões Centro-oeste $(7,4 \%)$ e Norte $(7,0 \%)$ foram as que tiveram os menores índices de municípios atendidos dentre os 517 alcançados em todo País. A FIGURA 1 mostra a distribuição espacial dos alunos concluintes dos cursos de capacitação oferecidos pelo Programa. Observa-se nesta figura, uma maior concentração de profissionais na Região Centro-Sul do país, regiões mais desenvolvidas como o Litoral e as capitais, mas também observamos alguns pontos nas regiões do interior do País e uma pequena concentração na região Norte, principalmente nos Estados do Amapá, Acre e Roraima.

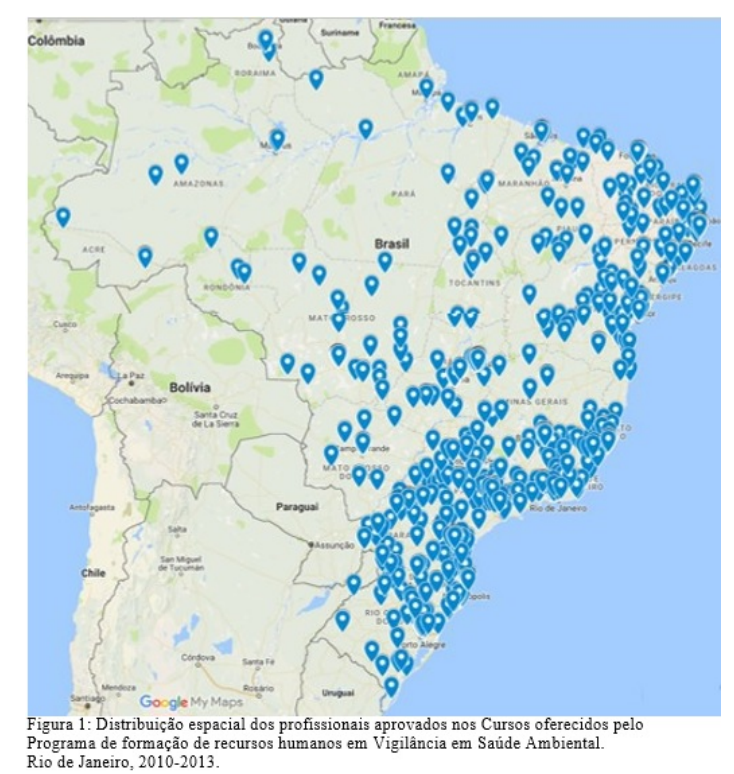

Após o envio de1894 questionários para 1.604 profissionais capacitados em dois momentos, no dia 05 de janeiro de 2017 e no dia 10 de fevereiro de 2017, para aqueles profissionais que não responderam no primeiro envio. Alunos que realizaram 2 ou os 3 cursos receberam um questionário específico para cada curso. No final, 33 e-mails enviados para os concluintes do Curso de Vigilância da qualidade da água para consumo humano, 48 para os concluintes do Curso de avaliação de risco e 44 para os profissionais do curso de Saúde, desastres e desenvolvimento retornaram automaticamente devido a algum problema no endereço eletrônico, caixa de mensagens cheia ou não localizada, restando 1479 e-mails com solicitação de resposta aos questionários anexados recebidos pelos profissionais. Do total de e-mails enviados (1479), 215 profissionais concordaram em participar da pesquisa, tendo sido respondidos $234(13,2 \%)$ questionários, visto que 3 indivíduos realizaram os três cursos de capacitação, 12 alunos realizaram dois cursos e 200 responderam apenas 0 questionário referente a um dos cursos. Dos 234 questionários respondidos, 91 (38,8\%) eram oriundos de alunos que finalizaram o curso de Avaliação de risco a saúde humana por exposição a substâncias químicas, 78 (33,3\%) do Curso de Vigilância da qualidade 
da água para consumo humano e 65 (27,8\%) do curso de Saúde, desastres e desenvolvimento e, significando uma taxa de resposta de 15\%, 13,6\% e $11 \%$, respectivamente, para cada curso em relação ao total de alunos capacitados por curso. Alunos de 127 municípios responderam ao questionário correspondendo a $24,6 \%$ dos 517 municípios do País com um ou mais profissionais capacitados nos cursos. Em relação ao aporte de informações que os cursos trouxeram para os profissionais da vigilância 231 (98,7\%) participantes responderam que de alguma forma, os cursos ampliaram suas competências em VSA. Os itens, ampliou totalmente e ampliou, escalas 4 e 5 da pergunta foram selecionados por 205 (88,7\%) profissionais (TABELA 3).

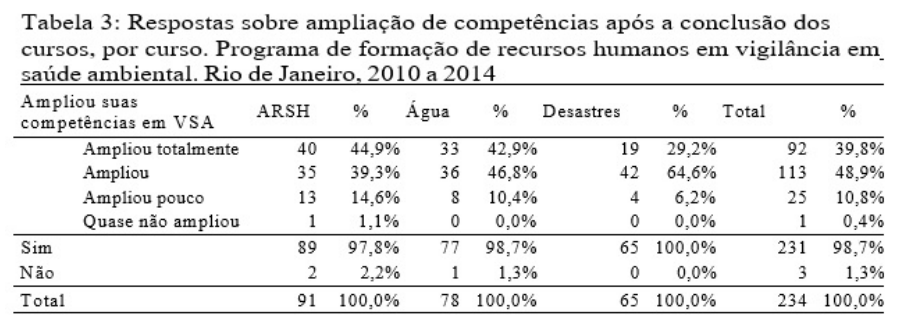

Os cursos de capacitação foram ofertados para profissionais de todo o Brasil. Com o objetivo de avaliar se a metodologia de educação a distância era uma boa estratégia de ensino na área de VSA, os alunos foram questionados a respeito das suas possibilidades de realizar um curso desta natureza no formato presencial. A maioria dos profissionais, $123(52,6 \%)$ respondeu que não conseguiria fazer os cursos caso estes fossem presenciais (TABELA 4). Adicionalmente, 219 (94\%) profissionais consideraram a experiência de realizar um curso à distância como ótima ou boa (TABELA 5).

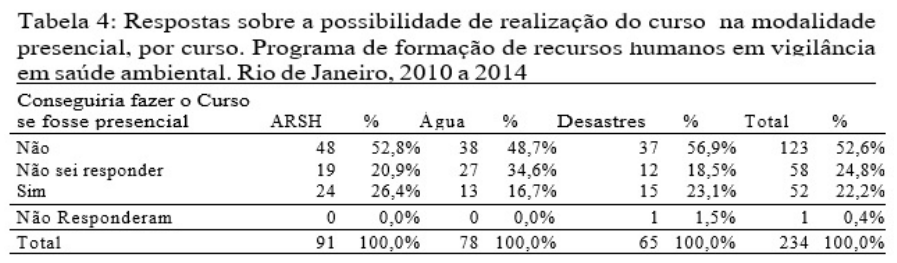

Tabela 5: Respostas sobre ạ experiência em EAD, por curso. Programa de formação de recursos humanos em vigilância em saúde ambiental. Rio de Janeiro, 2010 a 2014.

\begin{tabular}{lrrrrrrrr}
\hline Experiência em EAD & ARSH & \multicolumn{1}{c}{$\%$} & Água & \multicolumn{1}{c}{$\%$} & Desastres & \multicolumn{1}{c}{ Total } & $\%$ \\
\hline Otima & 50 & $54,9 \%$ & 52 & $66,7 \%$ & 37 & $56,9 \%$ & 139 & $59,4 \%$ \\
Boa & 35 & $38,5 \%$ & 23 & $29,5 \%$ & 22 & $33,8 \%$ & 80 & $34,2 \%$ \\
Regular & 6 & $6,6 \%$ & 2 & $2,6 \%$ & 5 & $7,7 \%$ & 13 & $5,6 \%$ \\
Ruim & 0 & $0,0 \%$ & 1 & $1,3 \%$ & 1 & $1,5 \%$ & 2 & $0,9 \%$ \\
Peśsima & 0 & $0,0 \%$ & 0 & $0,0 \%$ & 0 & $0,0 \%$ & 0 & $0,0 \%$ \\
\hline Total & 91 & $100,0 \%$ & 78 & $100,0 \%$ & 65 & $100,0 \%$ & 234 & $100,0 \%$ \\
\hline
\end{tabular}

4 - Discussão O alto percentual de alunos concluintes nos cursos ofertados pelo programa apresenta-se como um sinal positivo para o uso da metodologia de educação à distância na capacitação de profissionais. Estudos sobre conclusão e evasão de alunos em cursos de educação à distância têm relatado índices próximos a $50 \%$ (ABBAD, 2014; DE MORAIS, 2012), o que demonstra que o percentual de concluintes 
dos cursos ofertados pelo Programa (63\%) está acima do relatado na literatura sobre o tema. Cursos presenciais necessitam de maior deslocamento para os alunos, recursos financeiros para locomoção e alimentação, além de liberação do trabalho no período de realização do curso, o que pode inviabilizar a capacitação de muitos alunos em um mesmo período de tempo. O fortalecimento das ações de vigilância em saúde ambiental depende da capacitação dos profissionais que atuam nos serviços municipais, pois são estes que realizam em âmbito local as atividades da vigilância, atendendo aos problemas locais de cada região. Capacitando um maior número de profissionais que atuam em nível municipal, como observado nos resultados, capacita-se o profissional da ponta do serviço, contribuindo para a melhoria do conhecimento e consequentemente em suas decisões no enfrentamento das questões ambientais. Os questionários enviados aos profissionais concluintes dos cursos tiveram um percentual de resposta de $13 \%$, índice um pouco abaixo dos referidos pela bibliografia sobre as taxas de respostas em questionários online que é de $25 \%$ nos estudos de Vieira, Castro e Schuch Júnior (2010) e Almeida (2017) e de $21,3 \%$ no estudo de Kucharski e Basso, (2016). Uma possibilidade para esta taxa mais baixa pode ser discutida sob a perspectiva de que os cursos foram finalizados até o ano de 2014 e os questionários foram enviados no início de 2017. Este intervalo de tempo de no mínimo 3 anos pode ter coincidido com uma mudança de perspectivas profissionais, e mesmo pessoais, propiciando um desinteresse destes profissionais em participar da pesquisa. Profissionais dos estados de São Paulo e Rio de Janeiro foram os que mais responderam aos questionários e também foram os dois Estados com mais alunos capacitados nos cursos A maior capacitação destes profissionais e a maior adesão ao estudo podem estar relacionadas ao desenvolvimento das estruturas locais da vigilância em saúde ambiental. Quase a totalidade dos profissionais que responderam à pesquisa (98,7\%) afirmaram que os seus conhecimentos foram ampliados em algum grau em suas experiências com o curso online e 48,9\% relataram aquisição de maior competência para o desenvolvimento das ações de VSA. Ao citar os entraves que consideram atrapalhar o pleno desenvolvimento das atividades da vigilância em saúde ambiental, diversos problemas foram citados, principalmente os relacionados às questões políticas, recursos financeiros e falta de recursos humanos. A falta de estrutura física da vigilância em saúde ambiental, falta de interesse do gestor e a falta de capacitação das equipes também foram relatadas. Falta de comunicação entre os setores, excesso de trabalho, falta de condições de trabalho entre outras estão relacionadas a sobreposição de atribuições considerada como desvalorização das atividades de vigilância ambiental. Os entraves também foram identificados como dificuldades de integração com outras equipes, influenciando diretamente na distribuição dos recursos, na determinação das prioridades nas ações em saúde e consequentemente nas práticas das equipes de vigilância. Todas essas dificuldades são discutidas nos estudos sobre o desenvolvimento da área de vigilância 
em saúde ambiental nos três níveis de governo (TARTLER; PIRES; SANTOS, 2017; VILLARDI, et al., 2015; RECKTENWALDT; JUNGES, 2017). 5 - Conclusão A proposta da utilização da educação à distância para capacitação em grande escala dos trabalhadores da área da Vigilância em Saúde Ambiental é promissora como estratégia para consolidar e desenvolver as ações de fiscalização e controle das alterações no ambiente que podem de alguma forma interferir nas condições de saúde da população. O grande número de profissionais capacitados observados neste estudo mostra o quão positiva é esta estratégia para o desenvolvimento da área no País. Os cursos atingiram profissionais de diversas áreas do conhecimento, de todos os Estados e de quase 10\% dos municípios do país, fato que corrobora e justifica a utilização desta metodologia para capacitação profissional. É necessário evoluir nas questões políticas locais, na capacitação da equipe, na realização de concursos e na contratação de profissionais capacitados para o desenvolvimento das ações da vigilância ambiental. Esforços para melhoria na divulgação dos cursos de capacitação nas três esferas de Governo são necessários para podermos atingir mais profissionais em municípios mais distantes e assim desenvolver a área da vigilância em saúde ambiental no Brasil. Este é o caminho para uma vigilância mais atuante e que compreenda de forma integral as questões ambientais que possam causar o adoecimento da população e que atuem para prevenir e mitigar esses efeitos. 6 - Referências ABBAD, G. S. Educação a distância: o estado da arte e o futuro necessário. Revista do Serviço Público, v. 58, n. 3, p. 351-374, 2014. ALMEIDA, F. J. Permanência e abandono em cursos técnicos EAD no IFRS: enfrentamento do baixo índice de estudantes concluintes. 2017. 168 f. Dissertação (Mestrado Profissional em Gestão Educacional) - Universidade do Vale do Rio dos Sinos, Porto Alegre, 2017. AMÂNCIO, C. T.; NASCIMENTO, L. F. C. Asma e poluentes ambientais: um estudo de séries temporais. Rev Assoc Med Bras, v. 58, n. 3, 302-307, 2012. ASMUS, C. I. R. F. et al. A Systematic Review of Children's Environmental Health in Brazil. Annals of Global Health, v. 82, n. 1, p. 132-148, 2016. BECKER, J. L. Estatística Básica: Transformando Dados em Informação. Bookman Editora, 2015. BRASIL. Decreto no 3.450 de 9 de maio de 2000. Aprova o Estatuto e o Quadro Demonstrativo dos Cargos em Comissão e das Funções Gratificadas da Fundação Nacional de Saúde - FUNASA, e dá outras providências. Diário Oficial [da] República Federativa do Brasil, Poder Executivo, Brasília, DF, 10. mai. 2000. Seção 1, p. 1-5. CÂMARA, V. M.; TAMBELLINI, A. T. Considerações sobre o uso da epidemiologia nos estudos de Saúde Ambiental. Revista Brasileira de Epidemiologia, v. 6, n. 2, p. 95-104, 2003. DA SILVA et al. Programa de Formação de Recursos Humanos em Vigilância em Saúde Ambiental - Uma proposta de capacitação na área de vigilância em saúde. In: Relatos do uso de Tecnologias Educacionais na Educação permanente de profissionais da saúde no Sistema Universidade Aberta do SUS / Organização de Cristine Martins Gomes de Gusmão. [et al.]. — Recife: Ed. Universitária da UFPE, 2014. DE MORAIS, R. 
S.; VIANA, M. de L..F.; DE CAMARGO, R. A. A. Caracterização dos (as) estudantes de cursos de pós-graduação (Lato sensu) na modalidade de educação a distância. SIED: EnPED-Simpósio Internacional de Educação a Distância e Encontro de Pesquisadores em Educação a Distância 2012, 2012. FREIRE, P. Pedagogia da autonomia: saberes necessários à prática pedagógica. São Paulo: Paz e Terra, 1996. p. 165. GUIMARÃES, R. M. et al. $O$ impacto do consumo de agrotóxicos na prevalência de desfechos perinatais no Brasil. Boletín de Malariología y Salud Ambiental, v. 54, n. 1, p. 88-94, 2014. KRAWCZYK, N. et al. Revisiting cancer 15 years later: Exploring mortality among agricultural and non?agricultural workers in the Serrana Region of Rio de Janeiro. American Journal of Industrial Medicine, 2016. KUCHARSKI, M. V. S.; BASSO, V. H. Estudo da evasão no curso de especialização em tecnologias, comunicação e técnicas de ensino da UAB-UTFPR. Revista Intersaberes, v. 11, n. 23, p. 348-365, 2016. RECKTENWALDT, M.; JUNGES, J. R. A organização e a prática da Vigilância em Saúde em municípios de pequeno porte. Saúde e Sociedade, v. 26, n. 2, p. 367-381, 2017. ROHLFS, D. B. et al. A construção da Vigilância em Saúde ambiental no Brasil. Cadernos Saúde Coletiva, v. 19, n. 4, p. 391-398, 2011. TAMBELLINI, A. T; CÂMARA, V. M. A temática saúde e ambiente no processo de desenvolvimento do campo da saúde coletiva: aspectos históricos, conceituais e metodológicos. Ciência \& Saúde Coletiva, v. 3, n. 2, p. 47-59, 1998. TARTLER, N.; PIRES, H. H. R.; SANTOS, A. S. Avaliação do programa nacional de vigilância em saúde ambiental relacionada à qualidade da água para consumo humano no município de diamantina, minas gerais. Revista Desenvolvimento Social, v. 1, n. 20, p. 10, 2017. VIEIRA, H. C; CASTRO, A. E.; $\mathrm{SCHUCH}$ JÚNIOR, V. F. O uso de questionários via e- mail em pesquisas acadêmicas sob a ótica dos respondentes. In: SEMINÁRIO EM ADMINISTRAÇÃO-SEMEAD, 13., 2010. São Paulo, SP. Anais... São Paulo: USP, 2010. VILLARDI, J. W. R. et al. Planejamento de Recursos Humanos de Saúde e Ambiente- aspectos históricos, teóricos e reflexões para políticas públicas em Vigilância em Saúde Ambiental no Brasil. Tempus Actas de Saúde Coletiva, v. 9, n. 3, p. 105-120, 2015. 